\title{
General Collective Intelligence and the Cloud
}

\author{
Andy E. Williams ${ }^{1, *}$ \\ ${ }^{1}$ Nobeah Foundation, Nairobi, Kenya
}

\begin{abstract}
The resources that can be made available on-demand through cloud computing are continually increasing. One potential addition is General Collective Intelligence or GCI, which has been defined as a platform that combines individuals into a single intelligence with the potential for exponentially greater general problem-solving ability (intelligence) than any individual. The concept of a cognitive computing platform involves leveraging GCI to orchestrate cooperation between any entities that are required in order to create the capacity to maximize any collective outcome targeted. Rather than executing programming code, a cognitive computing platform must execute functional models in which each of the functional operations composing that model is implemented in some programming language. All services that run on the cloud, as well as the cloud itself, can potentially be offered as cognitive computing platforms. Where current cloud computing limits customers to a particular cloud service vendor, cloud computing as a cognitive computing platform has the potential to completely decouple users from any such dependencies, while at the same time creating the potential for an exponential increase in demand for cloud services from those vendors that participate by decoupling their services in this way.
\end{abstract}

Keywords: General Collective Intelligence, Human-Centric Functional Modelling, Artificial General Intelligence, cloud computing

\section{Background}

General Collective Intelligence or GCI [1], [2] has been defined as a platform that combines individuals into a single intelligence with the potential for exponentially greater general problem-solving ability (intelligence) than any individual. Part of this exponential increase might come from decomposing problems into a common functional model so they can be solved by a common set of cognitive computing operations that can be distributed over the whole group. By doing so GCI can be used to increase problemsolving ability by scaling the collective capacity to navigate complexity, by scaling the collective capacity to execute greater numbers of processes, and by scaling the ability to sustain processing through aligning processes to create greater

*Corresponding author. Email: awilliams@nobeahfoundation.org mutual value so that processing can become selfsustaining. Part of increasing these three capacities exponentially might come from employing intelligent agents incorporating some subset of Artificial General Intelligence (AGI) functionality, and using them to execute these operations on behalf of each user according to policies the user specifies, so that users can interact with the GCI at orders of magnitude greater speed and scale. In order for this interaction to occur at this greater speed and scale, it must be continuously occurring. For that to be the case, part of it must reside on a highly available platform like the cloud.

\section{Introduction}


Cognitive computing can potentially be applied to create cognitive applications in virtually any software domain, whether cognitive communications [3], the cognitive blockchain [4]. The functionality required by any cognitive computing platform is the functionality required for navigating conceptual space, the functionality required for executing those navigation operations with general problem-solving ability, the functionality required to decompose software and hardware into cognitive application domains, and the functionality required to scale cooperation between components of the cognitive system in executing the functionality in any application domain. If any application consists of a set of processes, and each of those processes consists of some sequence of activities, the functionality required to scale cooperation between components of the cognitive system in executing these processes is the functionality required to decouple the activities and the functionality to make that execution highly available.

\section{Related Work}

The functional modelling approach referred to in this paper has been used to define what is believed to be the first model of Artificial General Intelligence or AGI [5]. with the potential capacity for human-like general problem-solving ability, as well as a model for General Collective Intelligence or GCI [1], [2] with the potential for exponentially greater general problem-solving ability (intelligence) than any individual. Though others have described the concept of a general collective intelligence factor (c factor) [6], though at least one other has defined a model for a general collective intelligence factor [7], and though others have described the concept of a collective super intelligence [8], to the author's knowledge the author's own work is the only model that defines the specific mechanisms required for a GCI with the capacity to achieve super intelligence in that general collective intelligence factor. Because of this, and because this model is so new, all of the existing literature surrounding GCI refers to the author's own published work, or pre-prints of the author's unpublished work that is still under review.

\section{Research Questions and Approach}

The research questions addressed in this paper are approached with the method described below:

Section 4: Research Question Addressed

4.1 Can collective cognitive computing be applied to the cloud?

Section 5: Model

5.1. Software as automation.

5.2. Components of General Collective Intelligence on the cloud.

\section{Section 6. Method}

6.1. An analysis was performed to assess what core components are likely to be required.

6.2. An analysis was performed to assess whether the existence of similar functionality suggests the approach might be feasible.

6.3. An analysis was performed to assess how the cognitive computing platform model might be applied to an existing service.

6.4. An analysis was performed to assess whether the existence of similar functionality suggests the approach might be feasible.

Section 7. Findings

Section 8. Research Limitations

Section 9. Practical Implications

Section 10. Conclusions

\section{Model}

Any discussion of GCI and the cloud must begin with an understand of what GCI is executing, and what part of that is on the cloud.

\subsection{Software as Automation}

In the functional modeling approach that GCI is based on, any individual reasoning forms a path through conceptual space, and any collective reasoning forms a path through collective conceptual space. In this sense software applications are an automation of reasoning.

Since this semantic model is believed to have the capacity to represent all reasoning, if all software 
is an automation of reasoning then this model also has the capacity to represent the actions of all software. Semantic models restricted to a given functional domain are also functional models. On top of the basic semantic model, more convenient functional models might be constructed containing a set of operations spanning each application domain. Again, such functional models are also semantic models. These functions might be implemented in some programming language so that a GCI might orchestrate cooperation to select the best implementation in each context.

\subsection{Components of General Collective}

Intelligence on the Cloud

GCI orchestrates cooperation to execute applications that automate collective reasoning. There are three components to this process, the cooperation, the orchestration, and applications. The participants in the cooperation might be users in the physical world, or they might be intelligent agents interact with the GCI on the user's behalf. These agents can potentially reside on the cloud. The GCI orchestrating the cooperation can be on the cloud as well. The applications the GCI might interact with can be on the cloud as well.

\section{Method}

The research question was addressed using the methods summarized in table 2 .

Table 1. Research question and method.

\begin{tabular}{ll}
\hline Research Question & Method \\
\hline 1. Can collective & 1. Assess what core \\
cognitive computing & components are \\
be applied to the & likely to be required. \\
cloud? & 2. Assess whether \\
& the existence of \\
& similar functionality \\
& suggests the \\
& approach might be \\
& feasible. \\
\hline
\end{tabular}

3. Assess how the cognitive computing platform model might be applied to an existing service. 4. Assess whether the existence of similar functionality suggests the approach might be feasible.

\subsection{Required Functionality for Cognitive Computing Platforms on the Cloud}

The complete functionality required for cognitive computing platforms is the proposed Peer to Peer Social Fabric [9]. Some key functionality relevant to the cloud is described below.

\section{Decoupling the Physical Context of Execution}

Processes might be executed on any physical device the user owns, whether it be a smartphone, laptop, the cloud, or a dedicated supercomputer. Defining the actual device that a process executes on as the physical context of execution, then enabling processes to run on whatever physical hardware that becomes available, and then to switch processing to any better suited hardware that becomes available, requires defining a virtual context of execution as separate from the physical context of execution.

Since cognitive computing reduces computing to a common set of computing operations that might be executed on any suitably powerful hardware, defining a virtual context of execution spanning multiple physical devices is possible within this approach. Of course, this cognitive computing is based on a model of human cognition. Physical contexts of execution that lack the capacity to execute such cognitive operations would be confined to more simpler ones. An individual might have a large set of physical devices capable of executing cognitive computing operations. They might also have large swarms of devices, 
each capable of executing only a much simpler model of cognition. Only the first case is considered here.

\section{Decoupling the Ownership Related Context of Execution}

When a process is decoupled for collective execution, one of the properties of that process that must be decoupled is ownership. Ownership applies to the data provided as input to any activity in the process, the data produced as output by any activity in the process, and the data defining the process itself. All of this data must be represented in a common way (semantically). However, where other approaches to semantic modeling might have fallen short, this cognitive model is proposed to fulfill the requirements to enable the first complete semantic modeling functionality to be implemented.

Once data is modeled semantically so that it can be universally exchanged, ownership of that data must be decoupled from the context of execution so that each individual can engage in transactions to exchange ownership as they can participate in collective reasoning processes. Without decoupling ownership so that it can be decentralized to the individual, ownership is effectively centralized to the platform. The centralization of ownership of data is a fundamental bug (or feature) that prevents GCI from maximizing collective outcomes, because it ensures systems evolve in ways that serve the interests of the centralized owners.

\section{Decoupling Identity from Process Execution}

Another one of the properties of processes that must be decoupled for collective execution is identity. If an activity can be executed by a particular role, an activity might be executed by any individual in that role. If execution might take place on any physical context, then identity must be decouple from physical context as well.

\section{Putting Decoupled Contexts into the Cloud for Execution by Multiple Entities}

Having decoupled the physical context of execution, as well as having decoupled identity, ownership, and potentially other potentially non cloud-related properties of execution to be considered elsewhere, then these properties might be made portable to a universally available cloud platform so that processes can be executed collectively by users, by intelligent agents working on each user's behalf, or some combination of the two. In order for this to be possible, each collective cognition (GCI) application owned by some collection of individuals or other entities must run within an ownership context belonging to that set of entities, within a virtual physical context that executes on some physical device available to those users, potentially executing on the cloud. This GCI application will orchestrate the execution of activities by individual entities using software applications as tools to do so. These entities will execute these activities within an ownership context belonging to that entity, within a virtual physical context that again might potentially execute on the cloud.

The Peer to Peer Social Fabric (P2PSF) [9] is a proposed design for such a platform. While the functionality involved is broad and ambitious, using a GCI based approach to design enables needed components in existing products or services to be identified so they can be reused in other problems where they apply, so that solutions can potentially be developed at far greater speed and scale.

\subsection{Feasibility of Cognitive Computing Platforms on the Cloud}

A brief review of the literature was conducted to confirm that platforms providing a portable context of execution already exist, and are therefore feasible.

\subsection{Example: Required Functionality for a Cognitive Search Platform on the Cloud}

Assuming that a functional model can be defined to represent all the activities that can be performed with software in a given domain, then all activities through which users interact with a multi-user 
service in order to achieve some outcome might be represented in a swim lane diagram. Similarly, all if all activities in the service can be modeled, and if those activities can be decoupled so that each one can potentially be performed by a different provider, then the service those users interact with can be included on an expanded swim lane diagram.

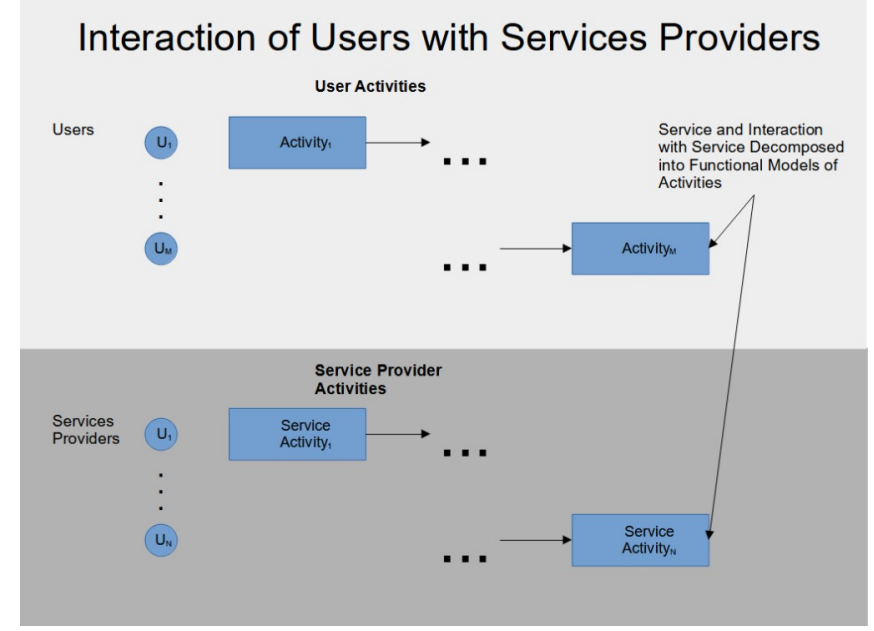

Figure 1. Activities involved in multi-user interactions with a service provided by multiple providers cooperating together.

In a cognitive application, a user interacts with an intelligent agent that incorporates some subset of the functionality required by an AGI. This intelligent agent executes a functional model of software in some application domain. The intelligent agent then selects the implementation of each function that is most fit in the current context. For example, such an agent might automatically change the user interface when executing on a smart phone as opposed to a personal computer.

Interaction of Users with Cognitive Applications in Context of Execution

User U,

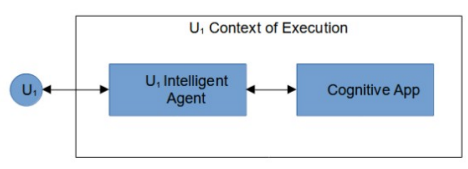

Figure 2. Cognitive computing applications have a virtual context of execution.

In order to facilitate the operation of a functional model based AGI or GCI, a platform such as the proposed Peer to Peer Social Fabric (P2PSF) must virtualize several properties. That is, it must decouple these properties from processes so these properties can be changed. The user's context of execution is a virtual property that might be implemented by any number of physical devices, or even be implemented by the cloud.

Physical Devices Implement User's Context of Execution

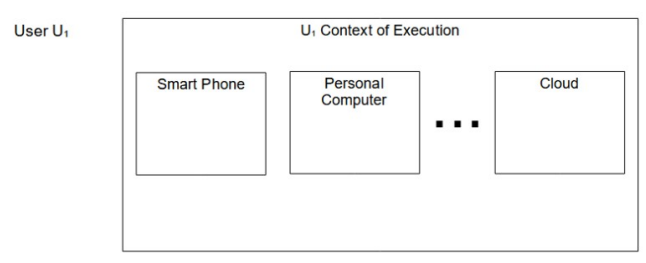

Figure 3. Many different physical environments might be within the user's context.

The context of execution of a user can be on the cloud, as can be the context of execution of a service provider offering a service through a cognitive computing platform.

\section{Contexts of Execution Deployed to the} Cloud

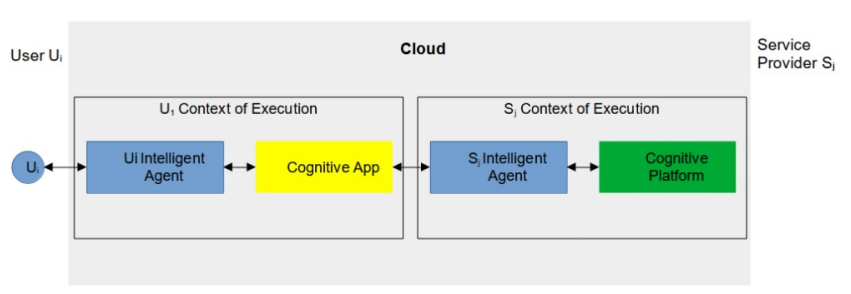

Figure 4. The virtual context of execution can potentially reside on the cloud.

As an example of how cognitive platforms deployed on the cloud might differ from current computing paradigms, consider the example of search. Current search engines are centralized in that they belong to a single provider Si. Search engines use bots to index each website Wi. Users then make search requests to the search engine and receive search responses. 


\section{Current Centralized Search Platforms}

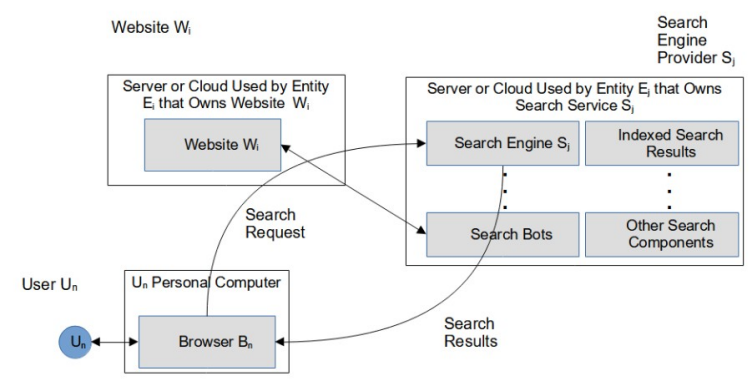

Figure 5. Current search engines with centralized ownership.

In a cognitive computing paradigm the user Un makes a request to an intelligent agent working on their behalf. This agent uses a cognitive app to make the request to a service provider $\mathrm{Sj}$. An intelligent agent working on behalf of the service provider indexes the website $\mathrm{Wi}$, receives the request, executes the search, and forwards the response.

Decentralized Search with a Cognitive Search Platform on the Cloud

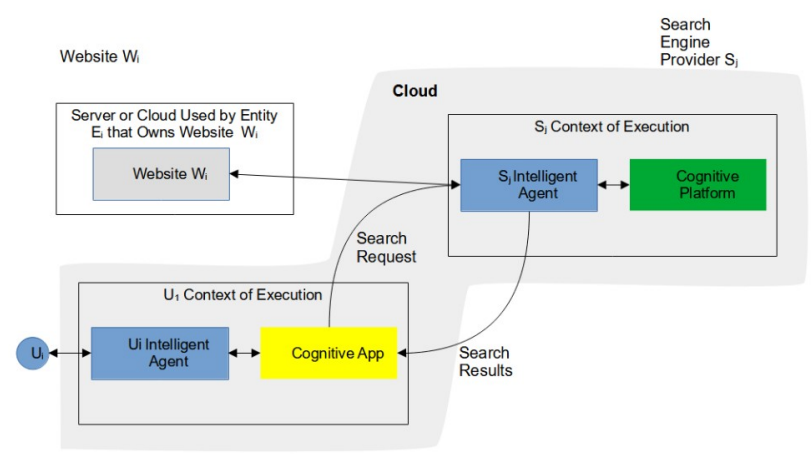

Figure 6. Cognitive computing based search on the cloud.

But since processes can be spread over multiple providers as in the swim lane diagram, it is possible to use a completely decentralized search protocol in which each entity requests the information from those in its network that are most likely to have it, who in turn ask those in their network who are most likely to have it, until a network self-assembles for that particular search. Because intelligent agents in each user's sole control respond to the search on their user's behalf, such searches could potentially be conducted at orders of magnitude greater speed and scale than any network of individuals could achieve without such agents.

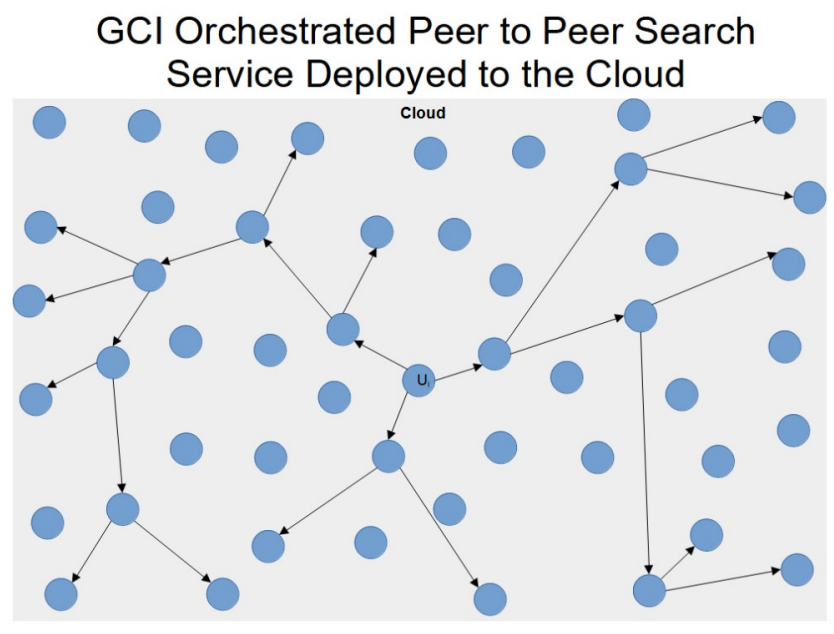

Figure 7. A decentralized, cognitive computing based search network.

In such a decentralized search protocol, each user can effectively be a service provider as well if the user context is put on a highly available cloud so these intelligent agents can continuously interact.

\section{User Node "i" in a Cognitive Search Platform on the Cloud}

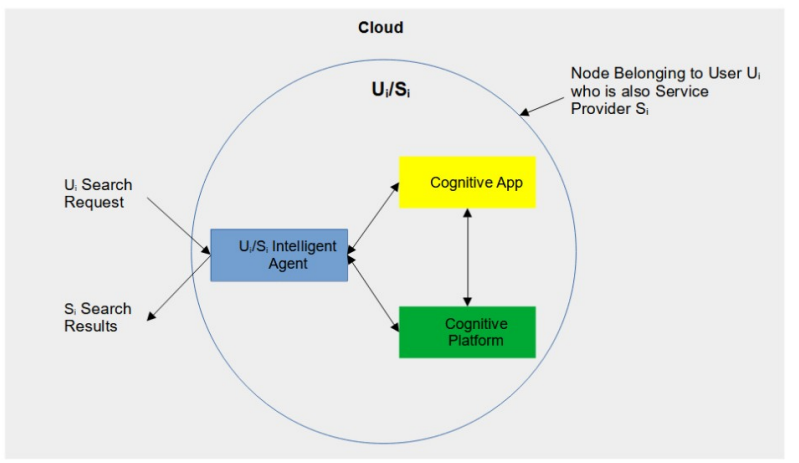

Figure 1. Each node in the search network might exist on the cloud.

6.4. Feasibility of a Cognitive Search Platform on the Cloud 
Many decentralized services already exist on the cloud.

A cognitive search platform would introduce a high degree of decentralization to the search process. A brief review of the literature was conducted to confirm that decentralized search platforms already exist, and that deploying a cognitive search platform on the cloud is therefore feasible.

\section{Findings}

The above analysis suggests that collective cognitive computing (i.e. cognitive computing platforms) can exist on the cloud. It also suggests that the cloud can become a cognitive computing platform itself. However, since GCI has not yet been fully implemented, many unknown hurdles remain to be crossed before this can be confirmed.

Table 2. Research Findings.

\begin{tabular}{ll}
\hline Research Question & Findings \\
\hline 1. Can collective & The answer \\
cognitive computing & appears to be yes \\
be applied to the & pending \\
cloud? & confirmation by an \\
& actual \\
& implementation. \\
\hline
\end{tabular}

\section{Research Limitations}

Neither GCI nor a cognitive computing platform have yet been completely implemented. As a result, this analysis has been conducted at a relatively abstract level. Actual implementation may reveal missing details.

\section{Acknowledgements.}

Thanks to Julian Talev for countless discussions on the topic.

\section{Practical Implications}

The implications of a vastly increased general collective intelligence factor are that a wide variety of seemingly intractable problems might become solvable, where not even solvable by supercomputers today. The implication of GCI on the cloud are that since GCI is predicted to exponentially increase capacity to impact any general problem where not predicted to be reliably achievable otherwise, and since the cloud is a fundamental requirement of GCI, then GCI might have the capacity to exponentially increase demand for the cloud where not reliably achievable otherwise.

One way that GCI might exponentially increase demand for the cloud is that GCI introduces the potential to individually customize every software product or service at an exponentially greater rate [10]. Applied to the Internet GCI introduces the potential to create in effect 7.5 billion Internet's, one for each person in the world. This involves a potentially massive increase in computing, storage, and other resources. However, GCI also has the potential to create sufficient value by aligning cooperation, perhaps by defining such configurations so they are optimally shared, so that financially supporting the deployment of such resources might be reliably achievable, and so that in turn such a vision is feasible.

\section{Conclusions}

General Collective Intelligence might have the capacity to exponentially expand the market for cloud services, but realizing that potential requires architecting products and services so they can be decoupled for execution by a GCI. This is a fundamental shift in architecture. It also requires implementing a GCI, which is a major effort in itself.

\section{References}

1 Conference: The Relationship Between Collective Intelligence and One Model of General Collective Intelligence, Andy E. Williams, Computational Collective 
Intelligence, 11th International Conference, ICCCI 2019, Hendaye, France, September 4-6, 2019, Proceedings, Part II, Pages 589600

2 Pre-print: Williams, A. E. (2020, December 2). General Collective Intelligence vs the Innate Collective Intelligence Factor. https://doi.org/10.31730/osf.io/kp3x8

3 Pre-print: Williams, A. E. (2020, September 24). Towards Cognitive Communication and Other Applications of Cognitive Computing. https://doi.org/10.31730/osf.io/fbh2p

4 Pre-print: Andy E. Williams, Roberto Moro visconti, Andy E. Williams, The Application of Artificial General Intelligence to the Cognitive Blockchain and the Internet of Value, DOI: 10.13140/RG.2.2.15004.31363

5 Book Chapter: Williams A.E. (2020) A Model for Artificial General Intelligence. In: Goertzel B., Panov A., Potapov A., Yampolskiy R. (eds) Artificial General Intelligence. AGI 2020. Lecture Notes in Computer Science, vol 12177. Springer, Cham. https://doi.org/10.1007/978-3-03052152-3 38

6 Journal article: Woolley, Anita Williams; Chabris, Christopher F.; Pentland, Alex; Hashmi, Nada; Malone, Thomas W. (29 October 2010). "Evidence for a Collective Intelligence Factor in the Performance of Human Groups". Science. 330 (6004): 686688. Bibcode:2010Sci...330..686W. doi:10.1126/science.1193147. PMID 20929725. S2CID 74579.

7 Journal article: Krafft, P.M. (2019), A Simple Computational Theory of General Collective Intelligence. Top Cogn Sci, 11: 374-392.

8 Book: Malone, T. W. (2018). Superminds: The surprising power of people and computers thinking together.

9 Pre-print: Williams, A. E. (n.d.). The Peer to Peer Social Fabric as a Platform for General Collective Intelligence. Retrieved from osf.io/preprints/africarxiv/qbxfr
10 Pre-print: Williams, A. E. (2020, December 16). Individualization of Products and Services with Artificial General Intelligence and General Collective Intelligence. https://doi.org/10.31730/osf.io/ gd5mt 